\title{
Management of Acanthamoeba and Candida Keratitis in a Young Female: Our Experience at Princess Haya Military Hospital
}

\author{
${ }^{1}$ Nancy Al Raqqad, ${ }^{2}$ Naser Al Fgara
}

\begin{abstract}
Aim: We aim to share our experience in the management of a 19 years old female patient, who presented to Princess Haya Military Hospital in Aqaba, Jordan, with a very aggressive keratitis.
\end{abstract}

Patients and methods: A 19 years old female patient with 1 year history of keratitis that did not resolve despite several treatment strategies tried elsewhere, presented to the eye clinic in Aqaba.

Ocular examination showed signs of Acanthamoeba keratitis with perineuritis.

Corneal cultures were not informative. Corneal biopsy showed a mixed Candida and Acanthamoeba growth.

Results: The patient was started on topical and oral antiamoebic and antifungal treatment (antiamoebic drops brought from UK). Improvement was drastic after 2 weeks of treatment. The patient maintained a chronic low infective state and scarring of the cornea. She received therapeutic and visual karatoplasty 3 months later.

Patient is now 12 months after her PKP. The cornea is clear and vision is $6 / 12$ unaided.

Conclusion: Cases of mixed fungal and amoebic keratitis are very rare. Prompt treatment and diagnosis is essential for recovery. Controversy still exists on the use of steroids after corneal transplantation for treatment of chronic fungal keratitis. Management should be tailored to each individual case.

Keywords: Acanthamoeba, Cornea, Keratitis.

How to cite this article: Al Raqqad N, Al Fgara N. Management of Acanthamoeba and Candida Keratitis in a Young Female: Our Experience at Princess Haya Military Hospital. Int J Kerat Ect Cor Dis 2015;4(3):120-122.

Source of support: Nil

Conflict of interest: None

\section{INTRODUCTION}

Acanthamoeba keratitis ( $\mathrm{AK}$ ) is a recently recognized infectious disease entity that is challenging to treat. It

\footnotetext{
${ }^{1}$ Consultant (Cornea and Refractive Surgery), ${ }^{2}$ Ophthalmologist

${ }^{1,2}$ Department of Ophthalmology, Royal Medical Services Amman, Jordan

Corresponding Author: Nancy AI Raqqad, Consultant (Cornea and Refractive Surgery), Department of Ophthalmology, Royal Medical Services, Amman, Jordan, Phone: 00962772080180 e-mail: nrakkad@yahoo.com
}

poses difficulties in diagnosis, treatment, management of associated complications and timing of surgical intervention.

Acanthamoeba spp. are a family of free-living cystforming protozoan that are ubiquitous in air, soil, dust, and water and can be isolated from the upper respiratory tracts of humans. ${ }^{1,2}$ In the cornea, it causes severe inflammation by feeding on keratocytes. ${ }^{1}$ The result is an aggressive keratitis, perinuritis, ring infiltrates and, if untreated, it can cause corneal ulceration and melting. Spread can also occur to the adjacent sclera and invasion to the anterior chamber.

Although occurs with higher incidence in soft contact lens wearers, still it is a differential diagnosis to consider in any patient with aggressive keratitis. Mixed infection, especially with herpes simplex keratitis, is not uncommon. 1,3

We report a case of a young female who was referred to our clinic after a prolonged history of unresolving severe keratitis.

\section{CASE REPORT}

A 19 years old female presented to our clinic on a Friday morning October 2012, complaining of severe eye pain, photophobia, epiphora and eye injection. History was traced back to 9 months before presentation. She was diagnosed of having keratitis and treated at a private hospital with fortified vancomycin and fortified fortum drops for 2 weeks. As her family saw no improvement, she moved from one ophthalmology service to another trying different kinds of medications with no signs of any improvement. She was given a cocktail of drops including antibacterial drops, antiviral ointments, and oral antiviral tablets with no use.

At one clinic, she was offered oral antifungal tablets for few weeks and started to feel slight improvement, yet the family chose to go to another practice and it was there that all her medications were stopped and she was offered a steroid-containing antibiotic drop. This time a severe flare up of her keratitis occurred and she was told that nothing can be done and poor prognosis was explained to the family. The patient dropped out of college. At this stage, we saw her in our clinic at Princess Haya Hospital in Aqaba. 
On examination, she had a best-corrected visual acuity (BCVA) of counting fingers 1 meter in her right eye, $6 / 6$ in her left eye. Slit-lamp examination showed severe circumcorneal injection, she was difficult to examine due to photophobia, corneal epitheliopathy, swollen corneal nerves (perineuritis) at 10, 11 and 1 clock hours. Examination also showed a $5 \mathrm{~mm}$ central corneal infiltrate covering $80 \%$ of the cornea, keratitic precipitates and starting vascularization.

Being a contact lens wearer and the presence of epitheliopathy and perinuritis put the diagnosis of AK on top of our differential diagnosis. Corneal cultures were taken in the clinic. She was kept on a broad spectrum fluoroquinolone drops hourly day and night. Meanwhile, we tried to provide her with antiacanthamoeba drops as soon as possible.

Three days later, the laboratory result came with no growth! The patient was taken to theaters and a $3 \mathrm{~mm}$ corneal biopsy was punched out. The disk was divided into two halves one was sent for histopathology and the other half for culture. The results came back within 72 hours positive for Candida albicans. And, few days later, the lab reported her specimen positive for acanthamoeba cysts.

Unfortunately, we did not find any antiacanthamoeba medications available in Jordan. And as her father was in United Kingdom at that time, we sent him a full report of her case so, he can get the drops. The report was taken to Moorfield's Eye Hospital in London and communication was made via telephone to dispatch PHMB (polyhexamethylene biguanide) and brolene drops after full discussion of the case.

The patient was started on oral fluconazole $150 \mathrm{mg}$ twice daily, amphotericin B $0.1 \%$ drops were prepared and were prescribed every hour day and night for the first 48 hours. ${ }^{1}$ The antiacanthamoeba drops came 10 days

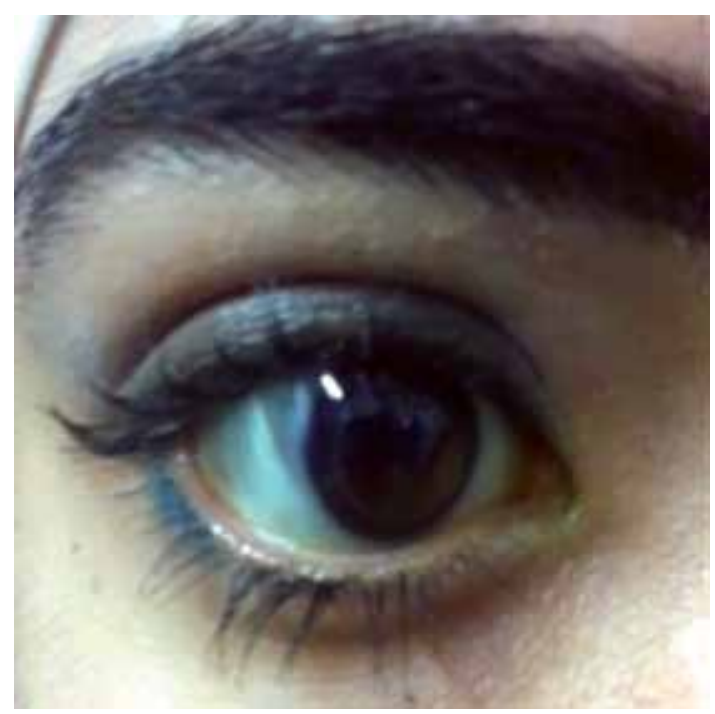

Fig. 1: Three months postpenetrating keratoplasty later and she was started immediately on PHMB 0.2\% and brolene hourly day and night for the first 48 hours, then shifted to hourly daytime only for another 72 hours then was maintained on 2 hourly drops for 3 to 4 weeks to avoid epithelial toxicity. She noticed improvement in her symptoms after 48 hours of treatment. The pain was less and the photophobia much better. Shrinkage in the size of the infiltrate by $50 \%$ was seen 2 weeks after initiation of treatment.

The duration of treatment was 4 months. She was maintained on PHMB and brolene, amphotericin B and oflox four times daily. Regression of the infiltrate and the vascularization was noticed. We, then, decided to list her for a penetrating keratoplasty.

On 14th January 2013, she received a penetrating keratoplasty. Washing the anterior chamber with amphotericin B was done. Sixteen 10.0 nylon sutures were used to secure an $8.25 \mathrm{~mm}$ corneal button into place. The next day challenge was to balance the steroid drops with her antifungal drops. She was started on pred forte drops four times daily along with her antifungal and antiacanthamoeba and antibiotic drops. She was seen every week to check for any sign of flare up vs rejection. Host button was sent for culture and histology and came back free of viable organisms. Four weeks later, her antibiotic drops were stopped, the PHMB and Brolene were kept to twice daily and the amphotericin B drops were stopped while she was maintained on oral fluconazole that was tapered over the following few weeks.

During her treatment, she underwent testing for her kidney function and liver function for any effect of the long-term use of fluconazole but all were normal.

Three months follow-up showed the patient's vision to be $6 / 12$ unaided, her graft was clear and she went back to college (Figs 1 and 2).

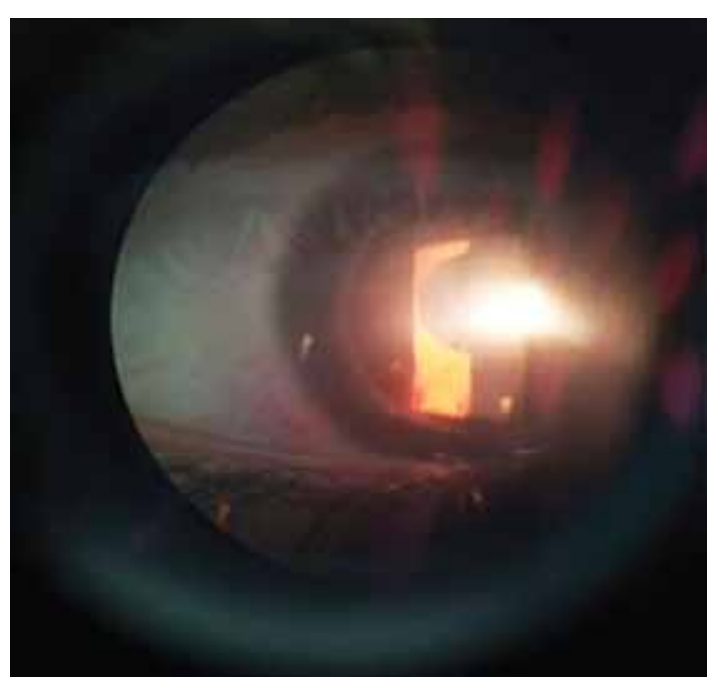

Fig. 2: Slit-lamp examination showing clear graft and quite eye postpenetrating keratoplasty 


\section{DISCUSSION}

Acanthamoeba keratitis is an underdiagnosed and difficult to treat infection of the cornea. ${ }^{1,4,5}$

Delayed diagnosis is associated with poorer prognosis as complications would be difficult to treat and organisms more resistant to kill.

Diagnosis can be made on detailed history, symptoms and clinical signs. The pain in AK is disproportionate to the physical signs. ${ }^{14-6}$

The incidence of mixed infection is 10 to $23 \%$ with herpes simplex keratitis being the most common, followed by streptococcal keratitis. ${ }^{1,2}$ Nevertheless, mixed infection with Candida albicans is very rare.

It poses a challenge on the treating physician not only in diagnosis but also in treatment as the introduction of steroid drops to minimize inflammation can also risk the flare up of the yeasts. At the same time, the prolonged use of various drops will result in more epithelial toxicity that might be mixed with worsening of the infection. ${ }^{\text {? }}$

The timing of the surgery is also another challenge for the ophthalmologist. More than $50 \%$ of AK patients will need a penetrating keratoplasty whether it is therapeutic, to rid the eye from a persistent infection, or visual to overcome scarring and rehabilitate vision. ${ }^{3,8}$ It is believed that early surgery carries a poor prognosis and endangers the eye and surgical intervention is advised for visual indications after the infection has been well under control.

The introduction of biguanides has dramatically improved success rate in the treatment of acanthamoeba keratitis. Although antibiotic and antifungal drops can kill the trophozoites, these are the cysts that are resistant to treatment, and, thus, biguanides are the mainstay of treatment of AK nowadays. ${ }^{1,8}$

\section{CONCLUSION}

Early recognition of $\mathrm{AK}$ is important for treatment. A positive history of contact lens wear, the presence of perineuritis and epitheliopathy all aid in diagnosis. Prompt treatment with biguanides can fasten recovery and avoid complications. Negative initial laboratory results can confuse and delay diagnosis. Treatment should be based on clinical judgment.

\section{REFERENCES}

1. Dart JK, Saw VP, Kilvington S. Acanthamoeba keratitis: diagnosis and treatment update 2009. Am J Ophthalmol 2009 Oct;148(4):487-499. e2.

2. Hammersmith KM. Diagnosis and management of Acanthamoeba keratitis. Curr Opin Ophthalmol 2006;(17)17:327-331.

3. Butler TK, Males JJ, Robinson LP, et al. Six-year review of Acanthamoeba keratitis in New South Wales, Australia: 1997-2002. Clin Experiment Ophthalmol 2005;33:41-46.

4. Clarke B, Sinha A, Parmar DN, Sykakis E. Advances in the diagnosis and treatment of acanthamoeba keratitis. J Ophthalmol 2012;2012: Article ID 484892.

5. McCulley JP, Alizadeh H, Niederkorn JY. The diagnosis and management of Acanthamoeba keratitis. CLAO J 2000 Jan; 26(1):47-51.

6. Wanachiwanawin D, Booranapong W, Kosrirukvongs P. Clinical features of Acanthamoeba keratitis in contact lens wearers and non-wearers. Southeast Asian J Trop Med Public Health 2012 May;43(3):549-556. Erratum in: Southeast Asian J Trop Med Public Health 2012 Jul;43(4):1065.

7. Panjwani N. Pathogenesis of acanthamoeba keratitis. Ocul Surf 2010 Apr;8(2):70-79.

8. Oldenburg CE, Acharya NR, Tu EY, Zegans ME, Mannis MJ, Gaynor BD, Whitcher JP, Lietman TM, Keenan JD. Practice patterns and opinions in the treatment of acanthamoeba keratitis. Cornea 2011 Dec;30(12):1363-1368. 